\title{
An AI Approach for Reducing Residual Motion and Noise from Myocardial Perfusion for Assessment of Coronary Artery Disease
}

\author{
Mahmud Hasan ${ }^{\dagger *}$ \\ ${ }^{\dagger}$ Department of Computer Science, University of Western Ontario
}

Keywords: low-dose CT, myocardial perfusion, deep learning, denoising, registration.

\section{Introduction}

Coronary Artery Disease (CAD) occurs when one or more coronary arteries become blocked or narrower, lowering the blood supply to the heart [1]. Analogous to stroke in the brain, this kind of blockage is also called myocardial ischemia. CAD is assessed (or scored) based on how much the arteries became narrower as compared to their average healthy size. Myocardial Perfusion Reserve (MPR) is the ratio of global myocardial blood flow at stress vs. rest. It is the theoretical gold standard technique for assessing myocardial ischemia in CAD [2]. Although myocardial perfusion can successfully detect and score the CAD, it often deals with some underlying problems due to the technology's nature, such as noise and residual motion. Myocardial perfusion imaging typically uses low-dose radiation as the myocardium is a radioactive sensitive organ by nature. But when the radiation is low, the constructed image will be noisy. Besides, in perfusion imaging, the images are taken over a period so that the contrast agent is visible in the images moving through the blood vessels. In such a setting, if the patient moves or the organ being scanned has motion itself (e.g. lung or heart), then the time point images do not entirely overlap, causing the blood vessels' positions inconsistent. This makes the assessment of perfusion parameters and CAD a challenging problem. As a result, a registered series of all-time points images is a must. By nature, this is a deformable registration problem because the heart (coronary region) has constant movement.

\section{Background}

Image denoising is the process of reducing noise or unwanted signals from images. Image registration is the process of aligning the images together that are misaligned due to organ or patient movement. We have studied a few learning-based denoising and registration methods where individuals are trying to solve these two problems independently [3-8]. But it is essential to jointly look at these two issues together instead of dealing with each one separately. A few reasons are supporting this heuristic. First, albeit the difference in their nature, both denoising and registration are optimization problems. Second, when problems are solved using Convolutional Neural Network (CNN) or other similar networks, different data sets are needed to solve various problems. Ideally, myocardial perfusion studies are a good option where a system can be trained at the same time using low-dose noisy CT data and the residual motion as these studies have features for both problems. Third, if we sequentially deal with both problems as in Lukas et al. [9], we will lose some valuable image information during denoising. Particularly for perfusion studies, since we also need the registration, we never know whether the information we are losing during denoising will be crucial to perform the registration. Therefore, it will be advantageous to solve both problems together. To the best of our knowledge so far, we did not find any

${ }^{*}$ mhasan62@uwo.ca

This article is (C) 2021 by author(s) as listed above. The article is licensed under a Creative Commons Attribution (CC BY 4.0) International license (https://creativecommons.org/licenses/by/4.0/legalcode), except where otherwise indicated with respect to particular material included in the article. The article should be attributed to the author(s) identified above. 
work attempting to solve both denoising and registration problems together, especially for low-dose myocardial perfusion, other than Lukas et al. [9]. We will have two significant contributions as an outcome of this research: (1) successfully using AI/Deep Learning for solving two optimization problems (denoising and registration) together, saving both time and space and (2) applying that model for better assessment of CAD in low-dose myocardial perfusion $\mathrm{CT}$ studies as a real-life clinical application.

\section{Research Plan}

We are developing a deep learning model capable of reducing the noise from the low-dose $\mathrm{CT}$ images. The model is trained with the high-dose $(80 \mathrm{~mA})$ myocardial CT perfusion data from animal studies and then tested on low-dose $(20 \mathrm{~mA})$ data to observe its performance. The model is expected to learn noise modeling, which usually follows a Poisson distribution in the case of CT. The noise pattern in a Four-Dimensional CT (4DCT) structure, such as myocardial perfusion, depends on the timing for contrast and location/slice for XRay attenuation. Therefore we first divide the given training data set $(80 \mathrm{~mA})$ into time points and slices. Then we train our model based on both the time points and location of the slice. Although this makes the learning challenging, on the other hand, it gives us some advantages in terms of handling the registration problem, as solving the registration problem depends on the exact location and time points for the images. Primarily we are trying to design our model following the recent state-of-the-art deep learning-based image denoising model $D n C N N$ [10] with some exceptions as needed by our case. First, this $D n C N N$ is a generalized image denoising model trained by a learning noise model following the Gaussian distribution of natural images. In CT perfusion, however, due to photon emission's nature, we have different noise distribution. Second, this model does not need to take the time and position information into account, as it was designed for denoising natural images. For our case, however, we need to modify the architecture to accommodate our needs. Third, as we intend to train the model for both denoising and registration, we also tweak the model to accommodate the myocardium's residual motion learning.

One challenge in the proposed method is that we often do not have gold standard data in myocardial perfusion setting. However, since we know that the noise tends to follow Poisson distribution in CT and we have the high-dose-low-noise $80 \mathrm{~mA}$ data, we introduce some synthetic Poisson noise in this data and assume that the original $80 \mathrm{~mA}$ images are gold standard for the synthetic noisy $80 \mathrm{~mA}$ images.

\section{Conclusion}

This research's expected outcome is a deep learning-based model trained (and tested) for reducing noise introduced due to low-dose radiation in CT imaging and reducing the residual motion generated due to the organ's movement (heart) myocardial perfusion studies. Once the model is trained and tested on animal studies with satisfactory performances, we will expand the training and testing on real human studies as well. So it is expected that if the results are promising, this model will have real clinical applications.

\section{Acknowledgments}

I am grateful to to Dr. Mahmoud R. El-Sakka, Dept. of Computer Science, UWO for his valuable supervision and to Dr. Aaron So, Dept. of Medical Biophysics, UWO for providing me low-dose myocardial perfusion animal studies for my experiments. 


\section{References}

[1] P. A. McCullough. "Coronary artery disease". In: Clinical Journal of the American Society of Nephrology 2.3 (2007), pp. 611-616.

[2] I.-L. Huang, M.-T. Wu, C. Hu, G.-Y. Mar, T.-Y. Lee, and A. So. "Quantitative low-dose rest and stress CT myocardial perfusion imaging with a whole-heart coverage scanner improves functional assessment of coronary artery disease". In: IJC Heart \& Vasculature 24 (2019), p. 100381.

[3] V. Wang, A. Wei, J. Tan, S. Lu, W. Cao, and Y. Gao. "A comparison study of deep learning designs for improving low-dose CT denoising". In: Medical Imaging 2021: Image Processing. Vol. 11596. International Society for Optics and Photonics. 2021, 115962N.

[4] J. Gu and J. C. Ye. "AdaIN-Based Tunable CycleGAN for Efficient Unsupervised Low-Dose CT Denoising". In: IEEE Transactions on Computational Imaging 7 (2021), pp. 73-85.

[5] T. Bai, D. Nguyen, B. Wang, and S. Jiang. "Deep High-Resolution Network for Low Dose X-ray CT Denoising". In: arXiv preprint arXiv:2102.00599 (2021).

[6] T. Wang, Y. Lei, Z. Tian, M. Giles, J. D. Bradley, W. J. Curran, T. Liu, and X. Yang. "4D cone-beam CT deformable registration using unsupervised spatial transformation network". In: Medical Imaging 2021: Biomedical Applications in Molecular, Structural, and Functional Imaging. Vol. 11600. International Society for Optics and Photonics. 2021, 116001K.

[7] Y. Zhang, X. Wu, H. M. Gach, H. Li, and D. Yang. "GroupRegNet: a groupwise one-shot deep learning-based 4D image registration method". In: Physics in Medicine 8 Biology 66.4 (2021), p. 045030.

[8] B. Dahman and J.-L. Dillenseger. "Deformable US/CT Image Registration with a Convolutional Neural Network for Cardiac Arrhythmia Therapy". In: 2020 42nd Annual International Conference of the IEEE Engineering in Medicine 83 Biology Society (EMBC). IEEE. 2020, pp. 2011-2014.

[9] S. Lukas, S. Feger, M. Rief, E. Zimmermann, and M. Dewey. "Noise reduction and motion elimination in low-dose 4D myocardial computed tomography perfusion (CTP): preliminary clinical evaluation of the ASTRA4D algorithm". In: European radiology 29.9 (2019), pp. 45724582 .

[10] K. Zhang, W. Zuo, Y. Chen, D. Meng, and L. Zhang. "Beyond a gaussian denoiser: Residual learning of deep cnn for image denoising". In: IEEE transactions on image processing 26.7 (2017), pp. 3142-3155. 